Article

\title{
Sensitivity and Stability Enhancement of Surface Plasmon Resonance Biosensors Based on a Large-Area $\mathrm{Ag} / \mathrm{MoS}_{2}$ Substrate
}

\author{
Nak-Hyeon Kim ${ }^{1,+}{ }^{\mathbb{D}}$, Munsik Choi ${ }^{2,+}$, Tae Woo $\mathrm{Kim}^{3}$, Woong Choi ${ }^{4}$, Sang Yoon Park ${ }^{1}$ \\ and Kyung Min Byun 2,* \\ 1 Advanced Institutes of Convergence Technology, Seoul National University, Suwon 16229, Korea; \\ nh-kim@snu.ac.kr (N.-H.K.); yoonpark77@snu.ac.kr (S.Y.P.) \\ 2 College of Electronics and Information, Dept. of Biomedical Engineering, Kyung Hee University, \\ Yongin 17104, Korea; blue-sky031@khu.ac.kr \\ 3 School of East-west Medical Science, Kyung Hee University, Yongin 17104, Korea; tw1275@khu.ac.kr \\ 4 School of Advanced Materials Engineering, Kookmin University, Seoul 02707, Korea; \\ woongchoi@kookmin.ac.kr \\ * Correspondence: kmbyun@khu.ac.kr; Tel.: +82-31-201-3842 \\ + These authors contributed equally to this work.
}

Received: 8 March 2019; Accepted: 16 April 2019; Published: 21 April 2019

\begin{abstract}
Surface plasmon resonance (SPR) sensors based on a silver film suffer from signal degradation due to silver oxidation in aqueous sensing environments. To overcome this limitation, we fabricated the planar plasmonic substrate employing an atomic $\mathrm{MoS}_{2}$ layer on a silver surface. Successful production of a large-area $\mathrm{MoS}_{2}$ monolayer blocks the penetration of oxygen and water molecules. In addition, we theoretically and experimentally found that $\mathrm{MoS}_{2}$ layer on the silver film can improve the SPR sensitivity and stability significantly. In this study, the proposed SPR substrate has the potential to provide highly enhanced sensor platforms for surface-limited molecular detections.
\end{abstract}

Keywords: surface plasmon resonance; biosensor; $\mathrm{MoS}_{2}$ monolayer; oxidation; sensitivity enhancement

\section{Introduction}

A surface plasmon (SP) is an electron charge density wave that exists at the interface between a thin metal film and a dielectric and propagates along the surface of the metal film [1]. When the transverse magnetic (TM) polarized light is incident on the metal film at a specific angle, the momentum of the incident light becomes equal to that of the plasmon and resonance occurs under this condition, which is called surface plasmon resonance (SPR). The specific angle at which resonance occurs and the reflected light gets completely attenuated is the SPR angle. When biomolecules adhere to a metallic surface, the resonance angle changes in proportion to the concentration of the target analytes [2]. Since SPR biosensors have advantages such as sensitivity, quantitative response, rapid and label-free detection, they have been widely used in a variety of analytical research fields [3].

Gold is typically used as a SPR substrate material, due to its chemical stability and reliability. On the other hand, it has been known that surface plasmons propagating along a silver surface exhibit a longer penetration depth into dielectric than those supported by a gold film [4]. SPR biosensors based on a silver film produce a sharp SPR curve, which can provide high selectivity and sensitivity in SPR imaging detection. However, an inevitable problem associated with application of silver films in SPR biosensors is that silver is highly susceptible to oxidation [5]. In particular, the oxidation of silver film can be fatal when it is exposed to an aqueous medium. The silver oxide formed by the oxidation process can degrade the SPR signal and interrupt surface-limited biomolecular reactions. 
In order to avoid silver oxidation, several approaches using stable metallic or dielectric coatings have been proposed. For example, addition of thin gold overlayer can prevent a silver film from being oxidized. [6,7]. However, individual surface plasmons produced by gold and silver films may interfere with each other, leading to a notable sensitivity degeneration compared to the case of a conventional single metal film [8].

Since the discovery of the single atomic layer two-dimensional (2D) structure of graphene, it has been considered a potential candidate for the protective layer of SPR substrates [9-11]. The thickness of a single graphene layer is about $0.34 \mathrm{~nm}$ and molecules cannot pass through its ring structure due to the high electron density of the hexagonal rings [12]. Hence, graphene is impermeable to oxygen and effective for protecting metal surfaces against corrosion [13]. Moreover, the plasmonic effects of graphene have been demonstrated for biological and chemical sensing applications both in the theoretical and experimental studies $[14,15]$.

Recently, monolayers of molybdenum disulfide $\left(\mathrm{MoS}_{2}\right)$ that belongs to the transition-metal dichalcogenides (TMDC) have been gaining great attention. The 2D structure of $\mathrm{MoS}_{2}$ is stacked in the vertical direction via Van der Waals forces $[16,17]$. As a monolayer of $\mathrm{MoS}_{2}$ possesses a higher optical absorption efficiency $(\sim 5 \%)$ than that of graphene $(2.3 \%)[18,19]$, it can promote plasmon excitation through an efficient charge transfer between $\mathrm{MoS}_{2}$ and the thin metallic film. However, despite its advantages, so far it has not been possible to successfully deposit a single layer of $\mathrm{MoS}_{2}$ uniformly on a large surface area. In this study, we fabricated $\mathrm{MoS}_{2}$ monolayers by chemical vapor deposition (CVD) and transferred them onto a large-area silver substrate. By comparing the sensor performance via non-specific binding experiments, we intend to demonstrate an enhancement of sensitivity and stability of SPR substrate with no concerns about oxidation.

\section{Materials and Methods}

\subsection{Fabrication of $\mathrm{MoS}_{2} / \mathrm{Ag}$-Based SPR Sensor Substrate}

Figure 1 shows the fabrication processes of the $\mathrm{Ag} / \mathrm{MoS}_{2}$-based SPR substrate. First of all, a sapphire glass (Schott, Mainz, Germany) and an NSF10 glass (Schott, Mainz, Germany) were prepared for the $\mathrm{MoS}_{2}$ transfer process. The glass substrates were cleaned by sonication with isopropyl alcohol for $10 \mathrm{~min}$, rinsed with deionized water for $10 \mathrm{~min}$, and dried with nitrogen gas for $10 \mathrm{~min}$. Then, a $\mathrm{MoS}_{2}$ layer was deposited onto the sapphire glass substrate using a CVD process. For $\mathrm{MoS}_{2}$ formation, $15 \mathrm{mg}$ of $\mathrm{MoO}_{3}(99.98 \%$, Sigma-Aldrich, St. Louis, MO, USA) and $1 \mathrm{~g}$ of S (99.98 \%, Sigma-Aldrich, St. Louis, $\mathrm{MO}, \mathrm{USA})$ powders were used as precursors.

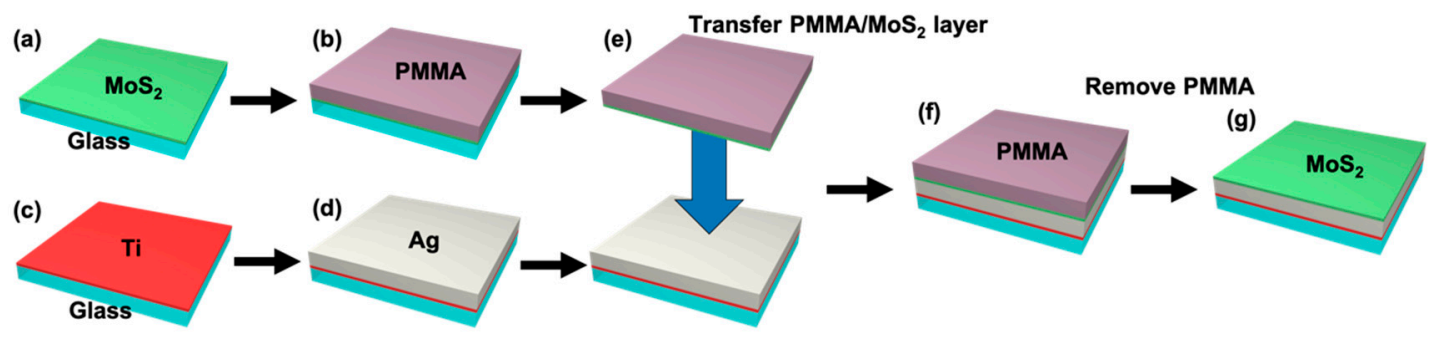

Figure 1. Schematic of fabrication processes of the $\mathrm{Ag} / \mathrm{MoS}_{2} \mathrm{SPR}$ sensor substrate. (a) $\mathrm{MoS}_{2}$ deposition using CVD, (b) PMMA spin-coating, (c) separation of $\mathrm{PMMA} / \mathrm{MoS}_{2}$ from Sapphire glass using $\mathrm{KOH}$, (d) Ti/Ag deposition using e-beam evaporation, (e) $\mathrm{PMMA} / \mathrm{MoS}_{2}$ transferring to Ti/Ag substrate, (f) PMMA removal using acetone.

The precursors were put on zone 1 of the CVD chamber and the sapphire glass substrate was put on zone 2. Then zone 1 and zone 2 were heated to the temperatures of $700^{\circ} \mathrm{C}$ and $600{ }^{\circ} \mathrm{C}$, respectively, and the pressure of the CVD chamber was maintained below 0.5 torr during $30 \mathrm{~min}$ for $\mathrm{MoS}_{2}$ deposition. After that, the temperature of the CVD chamber was decreased slowly to room temperature [20]. 5-nm thick titanium and 45-nm thick silver layers were sequentially deposited with a deposition rate of 
$3 \AA / s$ onto the NSF10 glass substrate using an electron beam evaporation. The titanium layer acts as an adhesion layer for silver deposition on the NSF10 glass substrate. To transfer the $\mathrm{MoS}_{2}$ layer onto the silver film, PMMA was spin-coated onto the $\mathrm{MoS}_{2}$ layer. After PMMA deposition, the sapphire glass was removed using wet etching with $\mathrm{KOH}$ for $1 \mathrm{~h}$, and then PMMA/MoS${ }_{2}$ layers were transferred onto the silver film. Finally, the fabrication of $\mathrm{Ag} / \mathrm{MoS}_{2}$-based SPR substrate with a large area was completed through PMMA removal by using a wet etching process with acetone.

\subsection{Optical Setup and Experimental Methods}

Our SPR sensor system based on the Kretschmann configuration which consists of a polarized He-Ne laser (05-LHP-991, Melles Griot, Irvine, CA, USA), dual rotation stages (SR50CC, Newport, Irvine, CA, USA) with a wide scanning range of 30 to 80 degrees at a resolution of 0.002 degree, a semicircular prism (customized model, Korea Electro-Optics, Bucheon, Korea) and a photodiode (918D-SL-OD3, Newport).

We investigated the oxidation stability and sensor sensitivity of the fabricated SPR substrates. To demonstrate oxidation stability, $\mathrm{Ag} / \mathrm{MoS}_{2}$ and bare $\mathrm{Ag}$ substrates were exposed under slow and fast oxidation conditions. Fast oxidation experiments were performed when a TM-polarized laser with a $10 \mathrm{~mW}$ power at the wavelength of $633 \mathrm{~nm}$ was incident under resonance conditions. In addition, to compare the SPR sensing performances of the fabricated substrates, immunoglobulin G (IgG) from human serum (PN I4506, Sigma-Aldrich) was used as a target analyte. Since the $\mathrm{MoS}_{2}$ and Ag surfaces are chemically different, a strategy to attach a non-specific analyte based on physisorption binding to the surfaces of two sensor substrates was used. IgG $(200 \mathrm{uL}, 600 \mathrm{nM})$ dissolved in a pH 7.4 phosphate buffered saline (PBS) solution, was injected through the microfluidic channel for $10 \mathrm{~min}$ to attach analytes onto the substrates. The substrates were cleaned and rinsed with distilled water for $5 \mathrm{~min}$ and then, PBS (1 mL) was injected through the fluidic channel for $5 \mathrm{~min}$. The SPR signals were measured five times after each process in order to check reproducibility.

\section{Results and Discussion}

First, we confirmed that the $\mathrm{MoS}_{2}$ monolayer was successfully transferred to the silver substrate by analyzing the material composition of the fabricated substrate. While atomic force microscope (AFM) and energy dispersive X-ray spectroscopy (EDS) are generally used for thickness measurements and surface composition analysis, those methods cannot be used at the same time. On the other hand, Raman spectroscopy, which measures inelastic scattering photons generated by the unique vibrational spectrum of the molecule, can give us compositional information about the target sample. In addition, since the vibration mode of $\mathrm{MoS}_{2}$ changes as the number of $\mathrm{MoS}_{2}$ layers increases, the thickness can be estimated by analyzing its Raman signal. $\mathrm{MoS}_{2}$ has four types of vibrational modes, $\mathrm{E}^{2}{ }_{2 \mathrm{~g}}, \mathrm{E}_{\mathrm{g}}{ }^{1}, \mathrm{E}^{1}{ }_{2 \mathrm{~g}}$ and $A_{1 \mathrm{~g}}$. The wavenumber of each mode is $32 \mathrm{~cm}^{-1}, 286 \mathrm{~cm}^{-1}, 383 \mathrm{~cm}^{-1}$ and $408 \mathrm{~cm}^{-1}$. Note that, wavenumber gap between $\mathrm{E}_{2 \mathrm{~g}}^{1}$ and $\mathrm{A}_{1 \mathrm{~g}}$ can be used to find the number of $\mathrm{MoS}_{2}$ layers.

With an increment of the layer number of $\mathrm{MoS}_{2}$, the wavenumber of $\mathrm{A}_{1 \mathrm{~g}}$ mode, which is related with vertical vibration, is increased and the wavenumber of $\mathrm{E}_{2 \mathrm{~g}}^{1}$ mode, which represents vibration in the same plane, is decreased by means of Van der Waals forces and Coulombic interactions. As a result, the $\mathrm{MoS}_{2}$ monolayer has a wavenumber gap of $18 \sim 20 \mathrm{~cm}^{-1}$ and the multi-layered $\mathrm{MoS}_{2}$ has a wavenumber gap of $25 \mathrm{~cm}^{-1}$ or more [20]. Figure 2 shows the Raman spectra of the fabricated $\mathrm{Ag} / \mathrm{MoS}_{2}$ substrate. The observed Raman spectra include Raman peaks of $\mathrm{MoS}_{2}$ at $386 \mathrm{~cm}^{-1}$ and $406 \mathrm{~cm}^{-1}$, thus $\Delta=20 \mathrm{~cm}^{-1}$, implying that $\mathrm{MoS}_{2}$ monolayer was successfully transferred onto the silver substrate. In addition, no Raman peak of PMMA is found because PMMA was removed completely through wet etching process. 


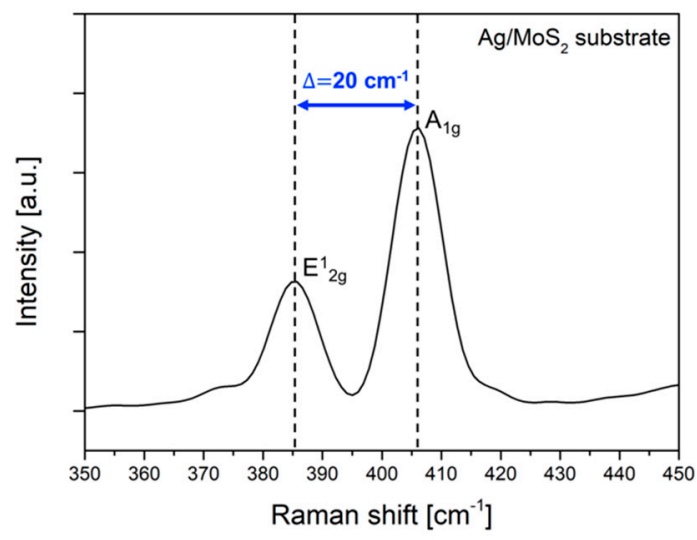

Figure 2. Raman spectra of the surface of $\mathrm{Ag} / \mathrm{MoS}_{2}$ substrate. To confirm the number of $\mathrm{MoS}_{2}$ layers, wavenumber gap between $\mathrm{E}_{2 \mathrm{~g}}^{1}$ and $\mathrm{A}_{1 \mathrm{~g}}$ mode is obtained. The measured Raman peaks are $386 \mathrm{~cm}^{-1}$ and $406 \mathrm{~cm}^{-1}$ (i.e., $\Delta=20 \mathrm{~cm}^{-1}$ ), supporting that the fabricated $\mathrm{MoS}_{2}$ layer on the $\mathrm{Ag}$ substrate is a single layer.

Next, we verified the role of $\mathrm{MoS}_{2}$ as a protective layer for silver film using slow and fast oxidation experiments. Figure $3 \mathrm{a}, \mathrm{b}$ present time-varying SPR curve changes for bare $\mathrm{Ag}$ and $\mathrm{Ag} / \mathrm{MoS}_{2}$ substrates in an aqueous solution. Since $\mathrm{Ag}_{2} \mathrm{O}$ is formed on the $\mathrm{Ag}$ surface as the oxidation progresses, the SPR signal becomes gradually broader according to the exposure time in the solution. However, the SPR signals of the $\mathrm{Ag} / \mathrm{MoS}_{2}$ substrate did not vary significantly for more than four days. The $\mathrm{MoS}_{2}$ layer consists of a sandwich structure of two $S$ layers and one Mo layer. These Mo and S layers have a hexagonal structure and $\mathrm{Mo}$ atoms are bonded with $\mathrm{S}$ atoms in a trigonal prism geometry. The $\mathrm{S}$ layers surrounding the Mo layer interacts with oxygen to give rise to high-energy barriers, which makes oxygen penetration into the $\mathrm{MoS}_{2}$ monolayer very difficult [21].

(a)

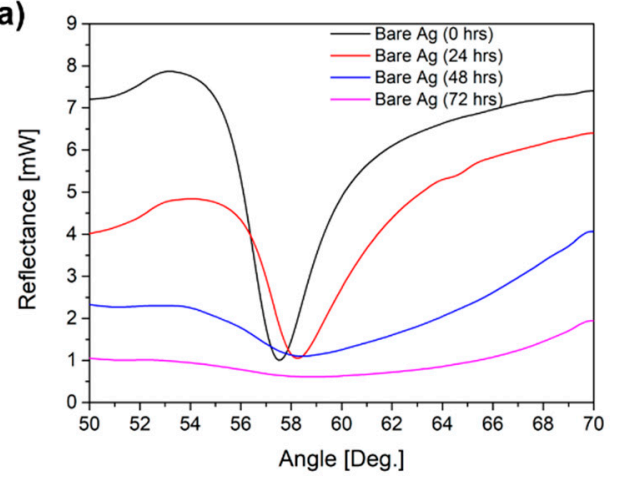

(b)

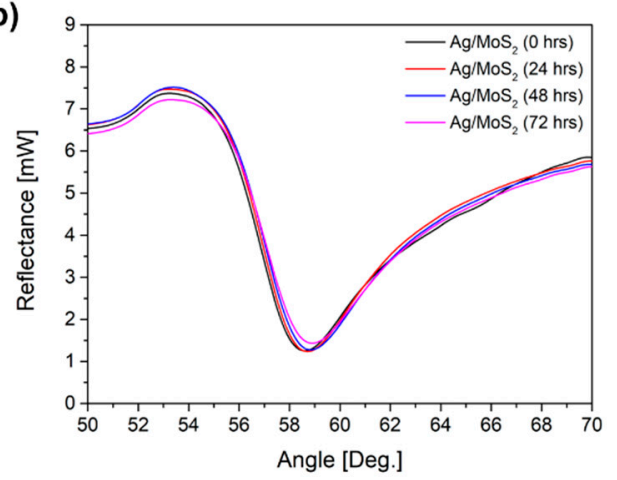

Figure 3. SPR signals of (a) bare $\mathrm{Ag}$ and (b) $\mathrm{Ag} / \mathrm{MoS}_{2}$ substrates in water. Silver oxidation may cause a broader SPR dip while $\mathrm{Ag} / \mathrm{MoS}_{2}$ substrate is stable due to impermeability to oxygen.

Figure $4 \mathrm{a}, \mathrm{b}$ show the SPR signal changes of bare $\mathrm{Ag}$ and $\mathrm{Ag} / \mathrm{MoS}_{2}$ substrates when they are irradiated by a laser in an aqueous solution. Due to temperature elevation via absorption of laser energy, faster oxidation process may occur. The bare Ag substrate in Figure 4a is oxidized as soon as it is exposed to a laser light. Moreover, as the obtained $\mathrm{Ag}_{2} \mathrm{O}$ on a silver film is photosensitive, the oxide layer decomposes when it is heated above the threshold temperature. It is found that formation of silver oxide and its decomposition can modify the SPR curves of the substrate drastically. On the other hand, the $\mathrm{Ag} / \mathrm{MoS}_{2}$ substrate does not change regardless of the laser irradiation, which means that $\mathrm{MoS}_{2}$ layer prevents the oxidation of silver and allows more stable features for application in practical SPR biosensors in aqueous medium. 
(a)

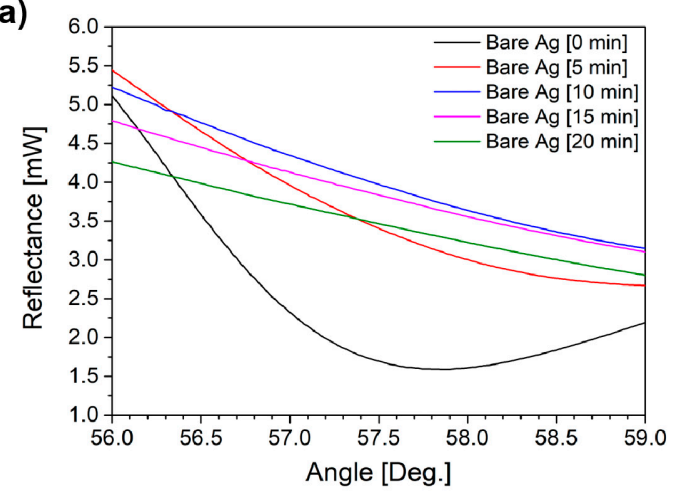

(b)

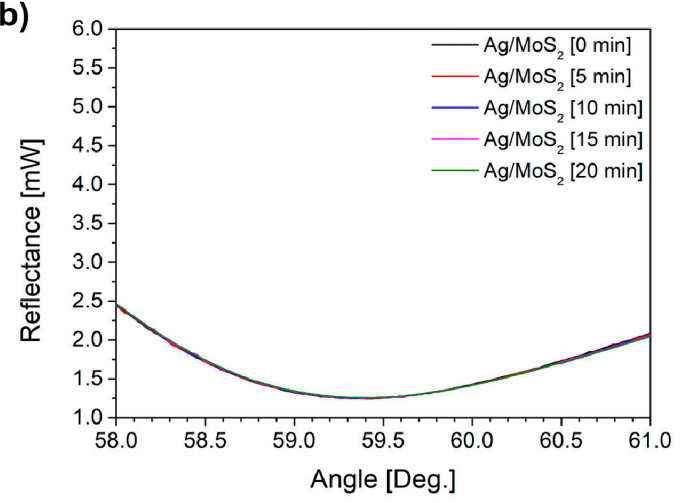

Figure 4. SPR signals of (a) bare $\mathrm{Ag}$ and (b) $\mathrm{Ag} / \mathrm{MoS}_{2}$ substrates in water with laser irradiation. Bare $\mathrm{Ag}$ substrate is easily oxidized as soon as it is exposed to a laser light and the SPR curves drastically change. Photosensitive $\mathrm{Ag}_{2} \mathrm{O}$, which is formed on the Ag surface during the progress of oxidation, decomposes when it is heated and the surface structure is damaged during the formation and decomposition of $\mathrm{Ag}_{2} \mathrm{O}$.

Figure 5 presents quantitatively the deformation of SPR graphs in Figures 3 and 4 according to the measurement time. The slope variation in Figure 5 means the variation of maximum slope value of individual SPR curves, i.e., the difference between the initial maximum slope value and the time-varying maximum slope value. The slope variation of the $\mathrm{Ag} / \mathrm{MoS}_{2}$ substrate is almost constant, however, that of the bare Ag substrate gradually decreases. This implies that a $\mathrm{MoS}_{2}$ layer confers enhanced stability to the substrate surface under slow and fast oxidization conditions.

(a)

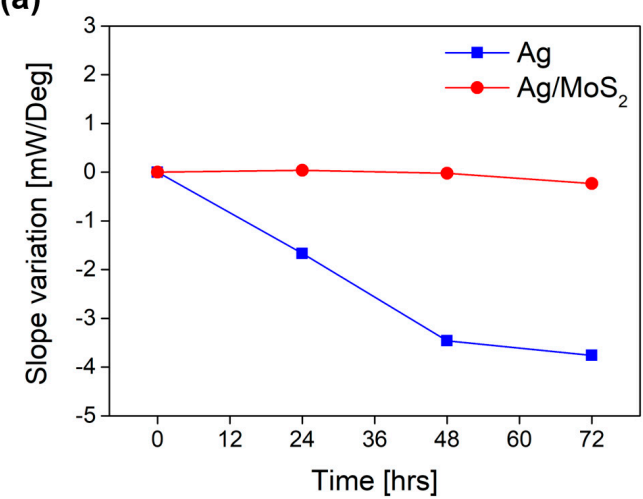

(b)

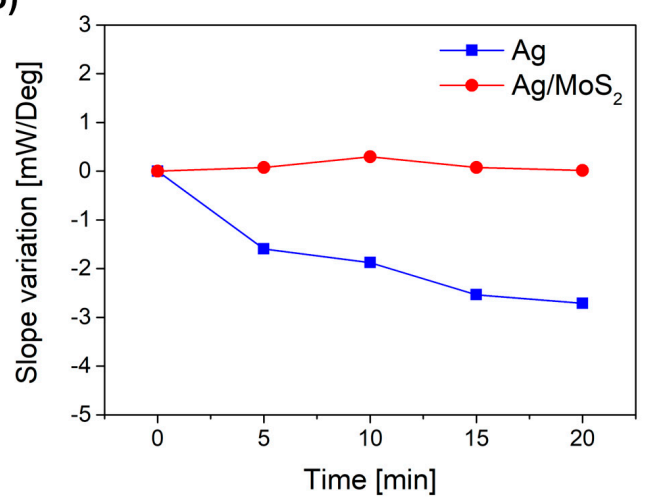

Figure 5. The slope variation of SPR signals of bare Ag SPR substrate (blue square) and Ag/MoS 2 SPR substrate (red circle) under (a) slow and (b) fast oxidation conditions. The slope variation of the SPR signal, which is defined as the intensity change of reflected light according to the angle, are represented according to measurement time.

In addition, to demonstrate the spatial uniformity of the $\mathrm{MoS}_{2}$ layer, SPR characteristics in multiple points are shown in Figure 6. The dashed line in the middle of the sample represents the area where $\mathrm{MoS}_{2}$ film is formed. It is presented in Figure $6 \mathrm{~b}$ that the SPR signals obtained at the center and four corners are very similar in shape. SPR angles and average value are summarized in the table in Figure $6 \mathrm{c}$. Considering the angle scanning resolution of 0.01 degree, we confirm that the $\mathrm{MoS}_{2}$ layer was uniformly deposited over the surface area. 

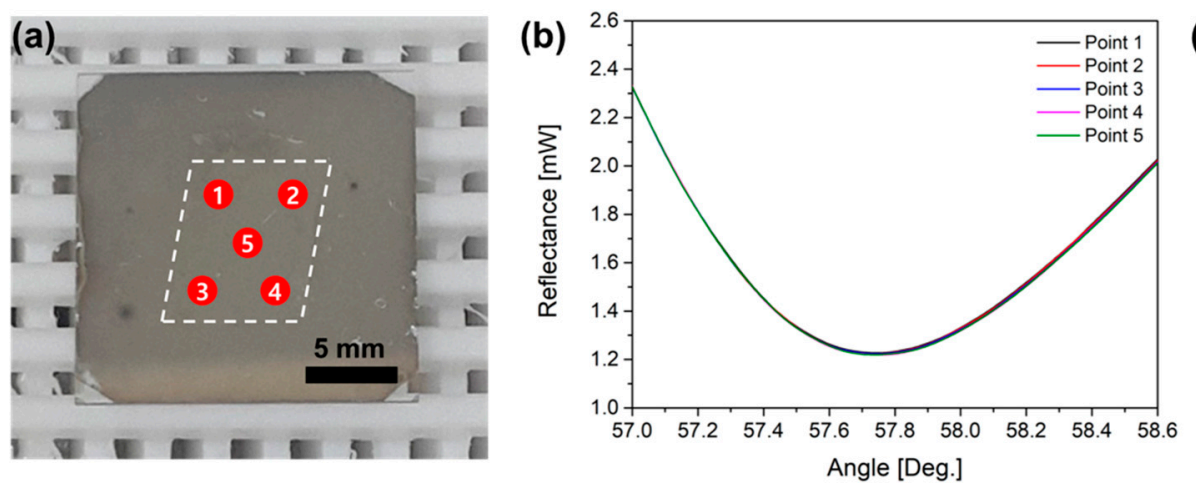

\begin{tabular}{|c|c|}
\hline Position & $\begin{array}{c}\text { SPR angle } \\
\text { (Deg.) }\end{array}$ \\
\hline Point 1 & $57.74^{\circ}$ \\
\hline Point 2 & $57.74^{\circ}$ \\
\hline Point 3 & $57.74^{\circ}$ \\
\hline Point 4 & $57.76^{\circ}$ \\
\hline Point 5 & $57.74^{\circ}$ \\
\hline Average & 57.744 \\
\hline $\begin{array}{l}\text { Standard } \\
\text { deviation }\end{array}$ & 0.009 \\
\hline
\end{tabular}

Figure 6. (a) The fabricated $\mathrm{Ag} / \mathrm{MoS}_{2}$ sample and five SPR measurement points in red circles. (b) SPR curves of the $\mathrm{Ag} / \mathrm{MoS}_{2}$ sample obtained at the five different positions. (c) Summary table of SPR angles and average value.

Finally, we compared the two types of SPR substrates in terms of sensor sensitivity. SPR signals of two chemically different $\mathrm{Ag}$ and $\mathrm{Ag} / \mathrm{MoS}_{2}$ surfaces are measured through physisorption binding of IgG at a single concentration of $600 \mathrm{nM}$. Note that sensor sensitivity can be obtained by computing the change in resonance angle of two cases of with and without binding of $\operatorname{IgG}$, while sensing experiments at different concentrations are possible only under chemical binding event conditions.

Figure 7a shows that the SPR angle shift of the $\mathrm{Ag} / \mathrm{MoS}_{2}$ substrate is larger before and after non-specific binding reaction of IgG than that of a bare Ag substrate. SPR angle shift of the bare Ag substrate is $0.20^{\circ}$ with a standard deviation (SD) of $0.008^{\circ}$ and that of the $\mathrm{Ag} / \mathrm{MoS}_{2}$ substrate is $0.25^{\circ}$ with SD of $0.010^{\circ}$, respectively. Together with an improvement of sensor sensitivity up to $125 \%$ by introducing a single $\mathrm{MoS}_{2}$ layer, it is expected that enhanced detection limit and responsivity are possible. It seems that the improved sensor performance is associated with the plasmon enhancement through high optical absorption efficiency of $\mathrm{MoS}_{2}$ layer and the promotion effect of the excitation process of an efficient charge transfer between $\mathrm{MoS}_{2}$ monolayer and thin metal film [22].

(a)

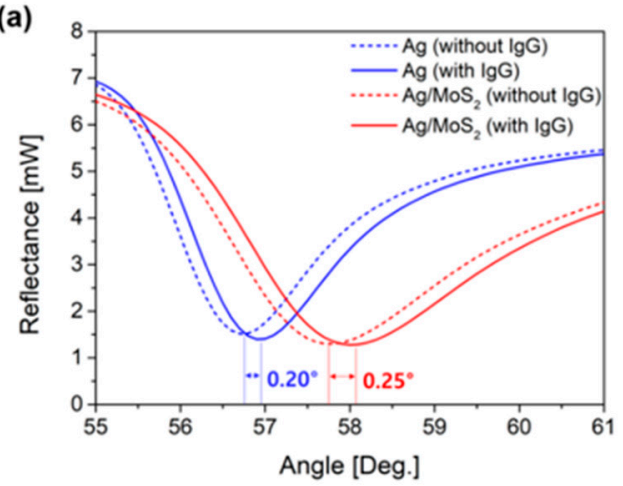

(b)

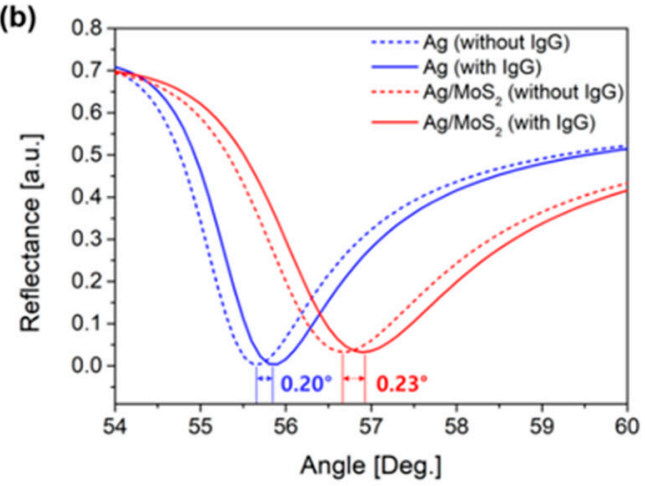

Figure 7. Experimental and simulation results of SPR signals for $\mathrm{Ag}$ and $\mathrm{Ag} / \mathrm{MoS}_{2}$ substrates for $\mathrm{IgG}$ detection. (a) SPR angle shift before and after the binding reaction of $\mathrm{IgG}$ of $\mathrm{Ag}$ substrate is $0.20^{\circ}$ and that of the $\mathrm{Ag} / \mathrm{MoS}_{2}$ substrate is $0.25^{\circ}$. (b) RCWA calculation result of the SPR angle shift is $0.20^{\circ}$ for a bare Ag substrate and $0.23^{\circ}$ for $\mathrm{Ag} / \mathrm{MoS}_{2}$ substrate.

In Figure $7 \mathrm{~b}$, we show the SPR angle shift of $0.20^{\circ}$ for a bare silver substrate and $0.23^{\circ}$ for $\mathrm{Ag} / \mathrm{MoS}_{2}$ substrate using RCWA calculation and the experimental results match the simulations consistently. The optical constants $\varepsilon=(n, k)$ of NSF10 glass, thin layers of titanium, silver, and $\mathrm{MoS}_{2}$ are set to be $(1.723,0),(2.047,3.164),(0.144,3.81)$, and $(5.9,0.8)$ respectively, at the wavelength of $633 \mathrm{~nm}$. Also, the refractive index of a phosphate buffered saline (PBS) solution is assumed to be 1.33. IgG binding reaction occurring at the surface of each sample is modeled as a $15 \mathrm{~nm}$ thick layer and the refractive 
index of the binding layer is set to be 1.35 which is determined by the effective-medium approximation according to the Maxwell-Garnett theory [23].

\section{Conclusions}

In this study, we fabricated a SPR substrate by incorporating an atomic $\mathrm{MoS}_{2}$ layer on top of a $\mathrm{Ag}$ film and demonstrated its enhancement of sensitivity and stability. In the SPR substrate based on Ag film, the exposed Ag layer was easily oxidized and $\mathrm{Ag}_{2} \mathrm{O}$ was decomposed by incident light in an aqueous environment. However, we found that when the $\mathrm{MoS}_{2}$ layer is introduced as a protective layer, the atomic $\mathrm{MoS}_{2}$ monolayer completely suppressed the oxidation of Ag film. The experimental results indicated that $\mathrm{MoS}_{2}$ monolayer can provide a reliable surface stability as well as an improved detection sensitivity. This seems to be attributable to the high energy barrier and high light absorption efficiency of the $\mathrm{MoS}_{2}$ monolayer. While the sensing experiment in this study is based on non-specifically bound analytes, in future research we will use a well-designed chemical binding event and the proposed $\mathrm{Ag} / \mathrm{MoS}_{2}$ substrate of high sensitivity and stability is expected to be applicable to the analysis of a variety of small target molecules.

Author Contributions: Writing-Original Draft Preparation, N.-H.K.; Writing-Review \& Editing, K.M.B.; Visualization, M.C.; Methodology, T.W.K.; Resources, W.C. and S.Y.P.

Funding: This research was funded by the National Research Foundation of Korea (NRF), grant number 2016M3A7B4910458, 2016M3A7B4910495, 2016R1A2B4014369, 2017R1D1A1B03035950, 2017R1A2B4012428.

Conflicts of Interest: The authors declare no conflict of interest.

\section{References}

1. Reather, H. Excitation of Plasmons and Interband Transitions by Electrons; Springer: Berlin, Germany, 1980.

2. Liedberg, B.; Nylander, C.; Lundstrom, I. Surface plasmons resonance for gas detection and biosensing. Sens. Actuators 1983, 4, 299-304. [CrossRef]

3. Shankaran, D.R.; Gobi, K.V.; Miura, N. Recent advancements in surface plasmon resonance immunosensors for detection of small molecules of biomedical, food and environmental interest. Sens. Actuators B Chem. 2007, 121, 158-177. [CrossRef]

4. Homola, J. Electromagnetic Theory of Surface Plasmons. In Surface Plasmon Resonance Based Sensors; Springer: Berlin, Germany, 2006; pp. 3-44.

5. Sahm, H.; Charton, C.; Thielsch, R. Oxidation behaviour of thin silver films deposited on plastic web characterized by spectroscopic ellipsometry (SE). Thin Solid Films 2004, 455-456, 819-823. [CrossRef]

6. Choi, S.H.; Byun, K.M. Investigation on an application of silver substrates for sensitive surface plasmon resonance imaging detection. Opt. Soc. Am. A 2010, 27, 2229-2236. [CrossRef] [PubMed]

7. Ong, B.H.; Yuan, X.; Tjin, S.C.; Zhang, J.; Ng, H.M. Optimised film thickness for maximum evanescent field enhancement of a bimetallic film surface plasmon resonance biosensor. Sens. Actuators B 2006, 114, 1028-1034. [CrossRef]

8. Wang, M.; Huo, Y.; Zhang, C.; Yang, C.; Ning, T.; Liu, X.; Li, C.; Zhang, W.; Man, B. Theoretical design of a surface plasmon resonance sensor with high sensitivity and high resolution based on graphene-WS2 hybrid nanostructures and Au-Ag bimetallic film. RSC Adv. 2017, 7, 47177-47182. [CrossRef]

9. Choi, S.H.; Kim, Y.L.; Byun, K.M. Graphene-on-silver substrates for sensitive surface plasmon resonance imaging biosensors. Opt. Express 2011, 19, 458-466. [CrossRef]

10. Kravets, V.G.; Jalil, R.; Kim, Y.-J.; Ansell, D.; Aznakayeva, D.E.; Thackray, B.; Britnell, L.; Belle, B.D.; Withers, F.; Radko, T.F.; et al. Graphene-protected copper and silver plasmonics. Sci. Rep. 2014, 4, 5517. [CrossRef]

11. Hong, H.Y.; Ha, J.S.; Lee, S.S.; Park, J.H. Effective Propagation of Surface Plasmon Polaritons on GrapheneProtected Single-Crystalline Silver Films. ACS Appl. Mater. Interfaces 2017, 9, 5014-5022. [CrossRef]

12. Jiang, D.E.; Cooper, V.R.; Dai, S. Porous graphene as the ultimate membrane for gas separation. Nano Lett. 2009, 9, 4019-4024. [CrossRef] [PubMed]

13. Bunch, J.S.; Verbridge, S.S.; Aiden, J.S.; van der Zande, A.M.; Parpia, J.M.; Craighead, H.G.; McEuen, P.L. Impermeable atomic membranes from graphene sheets. Nano Lett. 2008, 8, 2458-2462. [CrossRef] 
14. Zeng, S.; Sreekanth, K.V.; Shang, J.; Yu, T.; Chen, C.K.; Yin, F.; Baillargeat, D.; Coquet, P.; Ho, H.P.; Kabashin, A.V.; et al. Graphene-Gold Metasurface Architectures for Ultrasensitive Plasmonic Biosensing. Adv. Mater. 2015, 27, 6163-6169. [CrossRef]

15. Stebunov, Y.V.; Aftenieva, O.A.; Arsenin, A.V.; Volkov, V.S. Highly Sensitive and Selective Sensor Chips with Graphene-Oxide Linking Layer. ACS Appl. Mater. Interfaces 2015, 7, 21727-21734. [CrossRef]

16. Friend, R.H.; Yoffe, A.D. Electronic properties of intercalation complexes of the transition metal dichalcogenides. Adv. Phys. 1987, 36, 1-94. [CrossRef]

17. Lin, Y.C.; Dumcenco, D.O.; Huang, Y.S.; Suenaga, K. Atomic mechanism of the semiconducting-to-metallic phase transition in single-layered $\mathrm{MoS}_{2}$. Nat. Nanotechnol. 2014, 9, 391-396. [CrossRef] [PubMed]

18. Lopez-Sanchez, O.; Lembke, D.; Kayci, M.; Radenovic, A.; Kis, A. Ultrasensitive photodetectors based on monolayer $\mathrm{MoS}_{2}$. Nat. Nanotechnol. 2013, 8, 497-501. [CrossRef]

19. Thongrattanasiri, S.; Koppens, F.H.L.; de Abajo, F.J.G. Complete Optical Absorption in Periodically Patterned Graphene. Phys. Rev. Lett. 2012, 108, 047401. [CrossRef] [PubMed]

20. Baek, S.H.; Choi, Y.; Choi, W. Large-Area Growth of Uniform Single-Layer $\mathrm{MoS}_{2}$ Thin Films by Chemical Vapor Deposition. Nanoscale Res. Lett. 2015, 10, 388. [CrossRef] [PubMed]

21. Sen, H.S.; Sahin, H.; Peeters, F.M.; Durgun, E. Monolayers of $\mathrm{MoS}_{2}$ as an oxidation protective nanocoating material. J. Appl. Phys. 2014, 116, 083508. [CrossRef]

22. Zeng, S.; Hu, S.; Xia, J.; Anderson, T.; Dinh, X.Q.; Meng, X.M.; Coquet, P.; Yong, K.T. Graphene-MoS 2 hybrid nanostructures enhanced surface plasmon resonance biosensors. Sens. Actuators B Chem. 2015, 207, 801-810. [CrossRef]

23. Levy, O.; Stroud, D. Maxwell Garnett theory for mixtures of anisotropic inclusions: Application to conducting polymers. Phys. Rev. B 1997, 56, 8035. [CrossRef]

(C) 2019 by the authors. Licensee MDPI, Basel, Switzerland. This article is an open access article distributed under the terms and conditions of the Creative Commons Attribution (CC BY) license (http://creativecommons.org/licenses/by/4.0/). 